\title{
Estudio de la percepción sobre los factores institucionales que influyen en el rendimiento académico de estudiantes de la Universidad Estatal a Distancia de Costa Rica ${ }^{1}$
}

\author{
A Study of the Perception of the Institutional Factors That Influence the Academic \\ Performance of Students of the Distance State University of Costa Rica ${ }^{2}$
}

\section{Estudo da percepção dos fatores institucionais que influenciam o desempenho acadêmico de estudantes da Universidade Estatal a Distância da Costa Rica ${ }^{3}$}

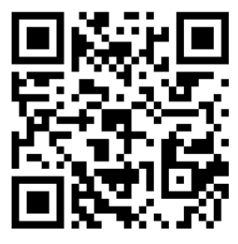

Corregido • Revised • Revisado: 24 / 03 / 2021

Aceptado • Accepted • Aprovado: 08 / 04 / 2021

José Alejandro Echeverría-Ramírez

Universidad Estatal a Distancia

San José, Costa Rica

jecheverria@uned.ac.cr

https://orcid.org/0000-0001-8896-4493

Claudia Mazzitelli

Universidad Nacional de San Juan

San Juan, Argentina

mazzitel@ffha.unsj.edu.ar

https://orcid.org/0000-0002-1199-4843

\begin{abstract}
Resumen:
Objetivo. Conocer la percepción estudiantil sobre la influencia de los diversos factores institucionales en el rendimiento académico, en una muestra de estudiantes de la Universidad Estatal a Distancia de Costa Rica (UNED), con el fin de contribuir con un análisis científico en los procesos formativos de educación a distancia. Metodología. El estudio se desarrolló desde un enfoque predominantemente de investigación cuantitativa. La muestra estuvo integrada por 418 estudiantes de la UNED de las regiones geográficas: Chorotega, Huertar Norte, Atlántica, Central, Brunca y Pacifico Central, lo anterior de acuerdo con la distribución vigente de las sedes que posee la universidad. Análisis de resultados. La recolección de datos se realizó por medio de una encuesta estructurada con un formato de escala Likert con cinco niveles de selección correspondientes a: me afecta mucho, me afecta poco, no me afecta ni me beneficia, me beneficia poco y me beneficia mucho. Para el análisis estadístico se empleó el programa SPSS. En este artículo se presentan los resultados obtenidos en relación con la percepción del estudiantado sobre la influencia de los factores institucionales en el rendimiento académico. Conclusiones. Los factores
\end{abstract}

\footnotetext{
${ }^{1}$ El artículo se deriva de la tesis doctoral, del Doctorado en Educación de la Universidad Internacional Iberoamericana, titulada Estudio de la percepción sobre los factores que influyen en el rendimiento académico de estudiantes de la Universidad Estatal a Distancia de Costa Rica.

${ }^{2}$ The article is derived from the Doctoral Thesis, from the Doctorate in Education of the Universidad Internacional Iberoamericana, entitled $A$ Study the perception on the factors that influence the academic performance of students of the State Distance University of Costa Rica.

${ }^{3} \mathrm{O}$ artigo é derivado da Tese de Doutorado, do Doutorado em Educação da Universidad Internacional Iberoamericana, intitulada Estudo da percepção sobre os fatores que influenciam o desempenho acadêmico de estudantes da Universidade Estadual a Distância da Costa Rica.
} 
http://doi.org/10.15359/ree.25-2.18

http://www.una.ac.cr/educare

educare@una.ac.cr

institucionales tales como: tipo de sistema de matrícula (matri-web), plataforma utilizada como entorno estudiantil y sistema de estudio a distancia, en la percepción del estudiantado, no influyen ni afectan el rendimiento académico en la UNED. El factor institucional de disponibilidad de cursos abiertos por cuatrimestre influye negativamente en el rendimiento académico de estudiantes de la UNED.

Palabras claves: Rendimiento académico; estudiantado universitario; factores institucionales; educación a distancia; percepción estudiantil.

\section{Abstract:}

Objective. To know the student perception about the influence of various institutional factors on academic performance, in a sample of students from the Distance State University of Costa Rica (UNED), in order to contribute with a scientific analysis in the distance education training processes. Methodology. The study was conducted from a predominantly quantitative research approach. The student sample was made up of 418 UNED students from these geographical regions: Chorotega, Huertar Norte, Atlántica, Central, Brunca, and Central Pacific, where the university has regional campuses. Analysis of results. The data collection was carried out through a structured survey with a Likert scale format with the following five levels of selection: it affects me much; it affects me little; it does not affect me or benefit me; it benefits me little: and it benefits me much. For the statistical analysis, the SPSS program was used. This article presents the results concerning the student's perception of the influence of institutional factors on academic performance. Conclusions. According to the student body's perception, institutional factors, such as the type of enrollment system (web-enrollment), the platform used as a student environment, and distance study system, do not influence or affect academic performance at UNED. The institutional factor of availability of open courses per semester negatively influences the academic performance of UNED students.

Keywords: Academic performance; University students; institutional factors; long distance education; student perception.

\section{Resumo:}

Objetivo. Conhecer a percepção de estudantes sobre a influência dos diversos fatores institucionais relacionados ao rendimento académico, com uma amostra de estudantes da Universidade Estatal a Distância de Costa Rica (UNED), com a finalidade de contribuir a uma análise científica nos processos formativos de educação a distância. Metodologia. O estudo foi desenvolvido a partir de uma abordagem de pesquisa predominantementequantitativa. Aamostra foiconstituída por 418 estudantes da UNED matriculados nas regiões geográficas: Chorotega, Huertar Norte, Atlântica, Central, Brunca e Central Pacifico, as anteriores de acordo com a atual distribuição da sede da universidade. Análise de resultados. A coleta de dados foi realizada por meio de uma pesquisa estruturada com formato de escala Likert com cinco níveis de seleção correspondentes a: me afeta muito, me afeta pouco, não me afeta e nem me beneficia, me beneficia pouco e me beneficia muito. Para a análise estatística, foi utilizado o programa SPSS. Este artigo apresenta os resultados obtidos em relação à percepção de estudantes sobre a influência dos fatores institucionais no desempenho acadêmico. Conclusões. Fatores institucionais como: tipo de sistema de inscrição (web-inscrição), plataforma utilizada como ambiente estudantil e sistema de ensino a distância, na percepção do corpo discente, não influenciam ou afetam o desempenho acadêmico na UNED. O fator institucional de disponibilidade de cursos abertos por semestre influencia negativamente o desempenho acadêmico dos alunos da UNED.

Palavras-chave: Rendimento acadêmico; estudantes universitários; fatores institucionais; Educação a Distância; percepção de estudantes. 
http://doi.org/10.15359/ree.25-2.18

\section{Introducción}

Las universidades públicas de Costa Rica enfrentan retos importantes a nivel nacional e internacional, por lo que el replanteamiento de una visión universitaria a futuro es necesario y este debe responder a las necesidades actuales de la población.

La planificación debe enfocarse y ser derivada de un proceso investigativo desarrollado en la población afectada, ya que una planificación sin fundamento científico trae como consecuencia una propuesta no viable o que no contribuye con las necesidades existentes, lo cual dificulta obtener los resultados esperados en el proceso formativo. De esta manera, el replanteamiento de la educación pública universitaria debe ser constante y fundamentado científicamente.

Uno de los retos más importantes para la educación universitaria costarricense es el rendimiento académico, el cual es influenciado por factores que dificultan o favorecen el proceso educativo y propician resultados variables en una población estudiantil. Así, aquellos factores que inciden en forma negativa pueden acentuar la deserción estudiantil, lo que será reflejado en los procesos de matriculación y en la cantidad de estudiantes que se gradúan.

La Universidad Estatal a Distancia de Costa Rica (UNED) es una institución de más de 40 años de antigüedad especializada en procesos de formación a distancia, debe generar acciones que permitan mejorar el rendimiento académico, para ello, en los procesos de planificación institucional debe propiciar espacios de investigación que recopilen insumos de la materia prima, es decir, del estudiantado.

La UNED debe evaluar los servicios que posee para conocer cómo estos afectan o benefician el rendimiento académico, con el fin de aumentar la cantidad de estudiantado graduado anualmente en relación con la matrícula ordinaria, pues este es uno de los retos más importantes que enfrenta la institución, al tener un promedio de graduación bajo en comparación con la matrícula.

Atendiendo a esto, la investigación buscó responder dos interrogantes principales: ¿Cuál es la percepción estudiantil sobre los factores institucionales, académicos y personales que influyen en el rendimiento académico? ¿Cuáles son los factores que más influyen en el rendimiento académico para estudiantes de la Universidad Estatal a Distancia de Costa Rica (UNED)? Es importante señalar que para este artículo solo se presentará el estudio de los factores institucionales.

Entre los objetivos planteados se pueden mencionar: conocer la percepción estudiantil sobre la influencia de los factores institucionales en el rendimiento académico, a fin de generar recomendaciones a la UNED para ser consideradas en los planes estratégicos venideros, y contribuir con el éxito académico del estudiantado.

En cuanto a los factores institucionales, se incorporaron dentro de la investigación como un mecanismo de conocer el impacto en el rendimiento académico de la inversión en infraestructura en la UNED, ya que este tipo de actividades universitarias poseen un constante cuestionamiento en la educación universitaria costarricense. 
http://doi.org/10.15359/ree.25-2.18

http://www.una.ac.cr/educare

educare@una.ac.cr

\section{Planteo del problema}

El rendimiento académico es una de las principales preocupaciones de las administraciones en los sistemas de educación superior de Costa Rica. En relación con el rendimiento académico exitoso, una forma práctica de detectarlo es por medio de la comparación de matrícula versus graduación, esto considerando el tiempo que dura una carrera universitaria.

Carpio Malavasi y Parreaguirre Camacho (2017), en el informe de labores 2017 de la Universidad Estatal a Distancia de Costa Rica, mencionan que la matrícula ordinaria de primer ingreso para el año 2011 fue de 10934 estudiantes (ver Figura 1).

Figura 1: Total de matrícula de estudiantes de primer ingreso del año 2011 al 2017

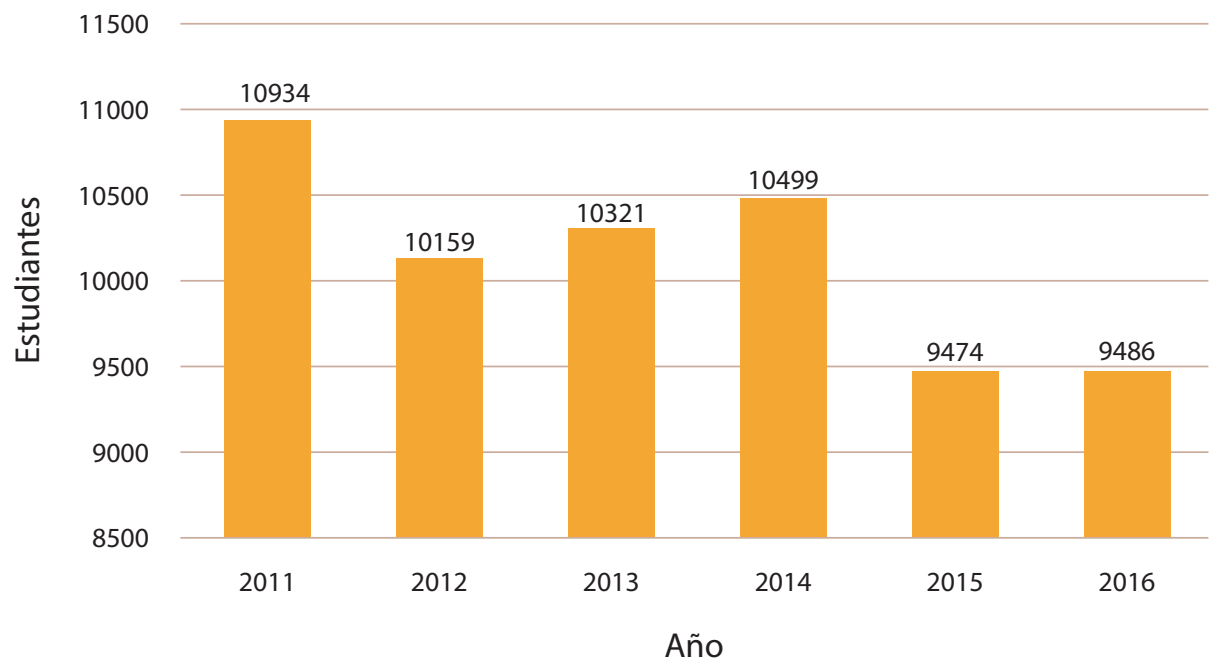

Nota: Elaboración propia a partir de información adaptada de Carpio Malavasi y Parreaguirre Camacho (2017).

Atendiendo a los planes deestudios de la UNED, el estudiantado debe obtener el bachillerato universitario al cuarto año de estudio, por lo que correspondería que se graduaran en el año 2015 quienes hayan ingresado en el año 2011, pero Carpio Malavasi y Parreaguirre Camacho (2017) mencionan que se entregaron 880 certificados en el grado de bachiller universitario en el año 2015, lo cual constituye solamente el 8\% de la matrícula del año 2011 (ver Figura 2). 
Figura 2: Total de graduados del año 2011 al 2017

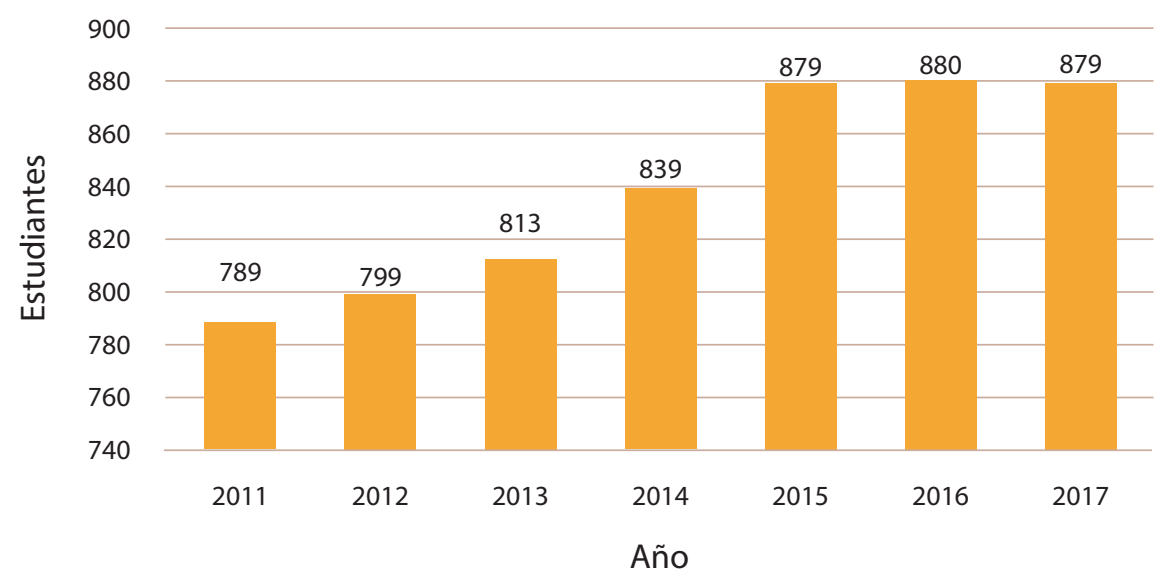

Nota: Elaboración propia a partir de información adaptada de Carpio Malavasi y Parreaguirre Camacho (2017).

Los datos indican que existen 10145 personas cursando estudios en la universidad que no lograron culminar su grado de bachiller en el periodo de los cuatro años al comparar la matrícula de primer ingreso 2011 versus las graduaciones de 2015. Es importante resaltar que no se poseen datos que indiquen si el estudiantado que consiguió culminar en el 2015 se encontraba rezagado en el sistema o pertenecía realmente a quienes habían hecho su primer ingreso en el 2011. Una situación similar se observa al comparar los valores de otros años (2012/2016, 2013/2017).

Según los datos mostrados anteriormente, se establece que el promedio de éxito académico ronda un $8 \%$ en la población de estudiantes de la UNED, esto se evidencia en la Figura 3.

Figura 3: Porcentaje total de graduados del año 2011 al 2017

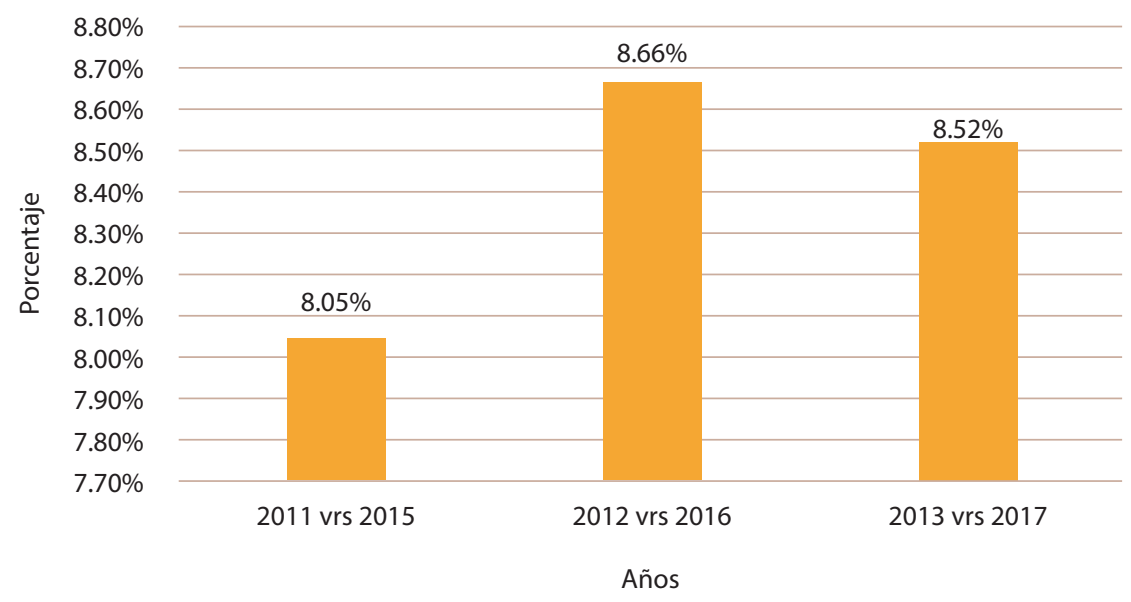

Nota: Elaboración propia a partir de información adaptada de Carpio Malavasi y Parreaguirre Camacho (2017). 
http://doi.org/10.15359/ree.25-2.18

http://www.una.ac.cr/educare

educare@una.ac.cr

Uno de los principales retos de la planificación para la UNED es poder generar acciones que mejoren el estado actual del rendimiento académico por medio del fortalecimiento de aquellos factores positivos y atención de los negativos. Para esto, es necesario, en primera instancia, identificar los factores que permitan lograr un mejor rendimiento académico a sus estudiantes y así reflejar mejores porcentajes de graduación por ciclo lectivo.

En cuanto a los factores institucionales que se analizaron fueron: Infraestructura, horario de atención, espacio físico, accesibilidad a internet, accesibilidad a tutorías y acceso a los laboratorios de informática en el centro universitario, horarios de atención de las tutorías, el lugar donde se imparten las tutorías, el sistema de entrega de tareas, la disponibilidad de cursos, el lugar donde se aplican los exámenes, el cronograma de aplicación de pruebas escritas, el sistema de matrícula web, los periodos lectivos cuatrimestrales, el horario de atención de la cátedra, el sistema de estudio a distancia, la información de la página web y plataforma.

\section{Referentes teóricos}

El rendimiento académico es un desafío que enfrentan estudiantes y docentes en todos los niveles educacionales. En los procesos de formación educativo intervienen una gran cantidad de variables que benefician o perjudican los procesos del estudiantado, entre ellas: la inseguridad social, dificultades con los contenidos, la falta de motivación, la descontextualización del currículo escolar, la carencia de interés por participar activamente en los procesos educativos de los miembros de la comunidad, entre otros.

Sánchez Herencia (2017) define al rendimiento académico como"medida de las capacidades del estudiante, que expresa lo que este ha aprendido a lo largo de todo su proceso formativo; también supone la capacidad del estudiante para responder a estímulos educativos" (p. 29).

Chilca Alva (2017) señala que "la dificultad de estudiar el rendimiento académico se inicia desde su definición, muchas veces se le denomina aptitud escolar, desempeño académico o rendimiento escolar, pero, generalmente, las diferencias de concepto solo se explican por cuestiones semánticas ya que se utilizan como sinónimos" (p. 74).

Al hablar de rendimiento académico en este trabajo es importante determinar e indicar que "los resultados de los alumnos constituyen el último nivel de análisis del funcionamiento de un sistema educativo" (Marchesi, 2000, p. 154). Para lograr un sistema educativo con equidad, es necesario proporcionar al estudiantado todas las herramientas educativas necesarias, y mantener un sistema funcional y eficiente. 
El rendimiento académico se encuentra entrelazado con la calidad educativa, Orozco Guzmán (2016, p. 19) expresa: "la calidad está concebida como la posibilidad de mejorar el ingreso económico de las personas a través de la adquisición de más conocimientos; de esta manera podrán incorporarse al mundo laboral para generar más ingresos".

De la Cruz (2009, p. 273) indica que "el estudiante permanezca en el proceso tiene que ver con dos aspectos: deserción y retención. En el primero se acostumbra a evidenciar los factores no institucionales que hacen que el estudiante se retire del proceso".

Aguila Cabrera (2005) realiza una reflexión en donde determina que la calidad no debe referirse exclusivamente a la evaluación externa y acreditación por parte de pares, sino que es un proceso de rendición de cuentas que debe estar acompañado de políticas que exijan la evaluación de la universidad. Al respecto afirma: "La discusión alrededor de la evaluación de la calidad de programas e instituciones universitarios ha pasado a planos superiores, ya no gira alrededor de si es necesaria y conveniente realizarla sino, ante todo, se relacionan con la búsqueda del cómo resulta más efectiva" (Aguila Cabrera, 2005, p. 1).

Por su parte, Pozón López (2014, p. 138) indica que "lograr una educación superior de calidad es un objetivo fundamental de cualquier sociedad. En este sentido se han llevado a cabo muchos esfuerzos por comprender y mitigar la deserción, por un lado, y por fomentar el aprendizaje y el buen desempeño académico de los estudiantes".

Así, el rendimiento académico es un desafío que enfrentan estudiantes y profesorado en todos los niveles educacionales. En los procesos de formación educativo intervienen una gran cantidad de variables que benefician o perjudican los procesos del estudiantado, entre ellos: la inseguridad social, dificultades con los contenidos, la falta de motivación, la descontextualización del currículo escolar, la carencia de interés por participar activamente en los procesos educativos de los miembros de la comunidad, entre otros.

Espinoza (2006) define el rendimiento académico como: una forma estimativa de lo que la persona ha aprendido en el proceso de formación dentro de una institución, a su vez determina que existen factores que influyen en estos resultados.

Garbanzo Vargas (2007) realiza un estudio en la Universidad de Costa Rica, en el cual investiga factores asociados al rendimiento académico y señala que en Costa Rica hay pocas investigaciones sobre este tema a nivel universitario.

Los diversos factores influyentes en concordancia con lo expresado por los estudios citados anteriormente son determinantes y tienen la fuerza de aumentar o disminuir los obstáculos 
http://doi.org/10.15359/ree.25-2.18

http://www.una.ac.cr/educare

educare@una.ac.cr

educativos. Por lo tanto, desde el accionar universitario es necesario implementar medidas que minimicen el abandono universitario, definido por Rojas (2009) como "un tipo de conducta con la cual el estudiante decide o es forzado a abandonar sus estudios" (p. 87).

Cabe señalar que los factores dependerán de la población y sus características únicas, las cuales delimitan el accionar debido a que las necesidades educativas son diversas, las cuales no pueden ser estandarizadas para toda la población educativa de un país, por ejemplo, un estudiante de secundaria no podrá ser comparado con una que se encuentre cursando una carrera universitaria, esto debido a que los factores de influencia son diferentes entre las poblaciones.

Atendiendo a los aportes analizados anteriormente, se hace evidente que existe una gran variedad de factores que influyen en el rendimiento académico, además de una clasificación o agrupación diversa.

Entre los factores institucionales mencionados por Garbanzo Vargas (2007) se encuentran: "la elección de los estudios según interés del estudiante, la complejidad en los estudios, las condiciones institucionales, los servicios institucionales de apoyo, el ambiente estudiantil, la relación estudiante -profesor y las pruebas específicas de ingreso a la carrera" (p. 57).

De esta manera se puede afirmar que los factores institucionales son los propios del complejo educativo que tienen una relación con los servicios brindados.

Es necesaria la identificación de estos factores que afectan o benefician el rendimiento académico tomando en consideración la percepción de su público usuario primario que, en este caso, corresponde al estudiantado. Al respecto, encontramos varios estudios vinculados a los factores que influyen en el rendimiento académico desde la percepción de estudiantes (Pineda Ledezma y Alcántara Galdámez, 2017; Vázquez et al., 2012; entre otros). La planificación institucional debe ser construida entre toda la comunidad universitaria, por lo que conocer la percepción del estudiantado a través de sus opiniones es fundamental en estos procesos de toma de decisiones.

\section{Metodología}

La investigación fue tipo no experimental, debido a que no hubo manipulación de las variables estudiadas, el fenómeno en estudio es una situación real que ocurre en la universidad, la población en estudio es preexistente en su entorno natural y los datos se recolectaron directamente para ser después procesados y analizados.

Este estudio fue descriptivo, señala la situación del estudiantado de la UNED por medio de la identificación de los factores que afectan el rendimiento académico en tres dimensiones: institucional, académico y personal. En este artículo solo se presenta el estudio en relación con los factores institucionales. 
La recolección de datos se realizó por medio de escala Likert con cinco niveles de selección: me afecta mucho, me afecta poco, no me afecta ni me beneficia, me beneficia poco, me beneficia mucho.

Esta investigación fue de carácter cuantitativo; además, en relación con la muestra, se ha realizado un muestreo probabilístico. El estudio puede considerarse exploratorio, porque no hay dentro de la UNED uno similar de acuerdo con la bibliografía y búsqueda documental realizada.

Los sujetos en estudios fueron estudiantes de la Universidad Estatal a Distancia de Costa Rica que se encontraban matriculados en un periodo continúo de al menos un cuatrimestre a la fecha de la aplicación de la encuesta.

Actualmente la UNED se encuentra conformada por 37 centros universitarios: San José, Heredia, Cartago, Palmares, Alajuela, Desamparados, Turrialba, Puriscal, San Marcos, Atenas, Acosta, San Isidro, Ciudad Neilly, San Vito, Buenos Aires, Osa, Liberia, Cañas, Nicoya, Santa Cruz, La Cruz, Tillarán, San Carlos, Upala, Sarapiquí, Pavón, Guápiles, Limón, Siquirres, Talamanca, Puntarenas, Orotina, Quepos, Monteverde y Jicaral, los cuales se encuentran distribuidos en diversas instalaciones a lo largo del territorio costarricense (Carpio Malavasi y Parreaguirre Camacho, 2017).

En lo correspondiente a infraestructura de los 37 centros universitarios, 17 poseen instalaciones propias y los otros 19 se encuentran localizados en lugares alquilados o espacios compartidos con centros educativos de primaria o secundaria. La UNED ha realizado varios esfuerzos por poseer, en su gran mayoría, instalaciones propias que se adecuen a las necesidades derivadas de las diversas instancias administrativas, académicas, de investigación, extensión, entre otras.

Tomando en consideración la información anterior, la investigación abordó todos los centros educativos para poder poseer una visión más real de la situación estudiantil en cuanto a su percepción de los factores institucionales y su impacto en el rendimiento académico.

Así, la extracción de la muestra de la población se realizó al azar, se tomó en consideración la matricula del último cuatrimestre del año 2017 por centro universitario de acuerdo con la región geográfica, exceptuando al estudiantado de la Reforma ya que estos poseen, por su condición de presidiarios, limitaciones para la aplicación de la encuesta y el estudio. Por lo que la población comprendió 1515 estudiantes (ver Tabla 1). 
http://doi.org/10.15359/ree.25-2.18

http://www.una.ac.cr/educare

educare@una.ac.cr

Tabla 1: Matrícula de estudiantes de primer ingreso del 2017 en el tercer cuatrimestre por centro universitario

\begin{tabular}{|c|c|c|c|c|c|}
\hline $\begin{array}{c}\text { Centros } \\
\text { Universitarios }\end{array}$ & $3^{\circ}$ Cuatrimestre & $\begin{array}{c}\text { Centros } \\
\text { universitarios }\end{array}$ & $3^{\circ}$ Cuatrimestre & $\begin{array}{c}\text { Centros } \\
\text { universitarios }\end{array}$ & $3^{\circ}$ Cuatrimestre \\
\hline San José & 332 & Acosta & 17 & Santa Cruz & 14 \\
\hline Heredia & 122 & San Isidro & 84 & La Cruz & 12 \\
\hline Cartago & 98 & Ciudad Neily & 39 & Tillarán & 8 \\
\hline Palmares & 79 & San Vito & 27 & San Carlos & 47 \\
\hline Alajuela & 109 & Buenos Aires & 33 & Upala & 32 \\
\hline Desamparados & 76 & Osa & 6 & Sarapiquí & 15 \\
\hline Turrialba & 50 & Puerto Jiménez & 1 & Pavón & 6 \\
\hline Puriscal & 38 & Liberia & 33 & Guápiles & 44 \\
\hline San Marcos & 20 & Cañas & 15 & Limón & 28 \\
\hline Atenas & 8 & Nicoya & 10 & Siquirres & 16 \\
\hline Monteverde & 0 & Orotina & 13 & Talamanca & 18 \\
\hline Jicaral & 8 & Quepos & 20 & Puntarenas & 37 \\
\hline Totales & 1515 & & & & \\
\hline
\end{tabular}

Nota: Elaboración propia a partir de información adaptada de Figueroa Retana y Gatgens Gutiérrez (2018).

En concordancia con la Tabla 1, la población del presente estudio está conformada por 1515 estudiantes ( $N=1515)$, para la extracción de la muestra se tomó en consideración el porcentaje de acuerdo con la matrícula por centro, en relación con la población total.

Para determinar el tamaño muestral, el nivel de confianza fue determinado en $95 \%$, con un error máximo permitido de $5 \%$, donde el tamaño de la muestra está siendo determinado por una probabilidad de ocurrencia de evento $p=0,50$, un margen de confianza $Z=1,96$, una probabilidad de que no ocurriera el evento de $q=0,50$, con un error máximo permitido de $E=0,05$.

El tamaño de la muestra fue de 418 estudiantes, realizado porcentualmente de acuerdo con la matrícula de referencia. 
http://doi.org/10.15359/ree.25-2.18

El instrumento se estructuró en cuatro apartados, pero para este artículo solo retomaremos los dos primeros que correspondieron a:

- Primer apartado: “Datos personales” (variables de identificación), en el cual se localizan 16 ítems que permiten recolectar información personal del encuestado (ver Apéndice A).

- Segundo apartado: "Opinión de estudiantes sobre los factores institucionales y el rendimiento académico", este comprende 18 ítems (ver Apéndice A) a través de los que se pretende recolectar información por medio de una escala Likert, sobre la percepción de la influencia de las variables institucionales en el rendimiento académico del estudiantado.

Como ya se adelantó, este instrumento se implementó por medio de una encuesta, para lo cual se llevaron a cabo los siguientes pasos:

- Se realizó una reunión en la que estuvieron presentes cuatro estudiantes de los centros universitarios de Alajuela, Heredia, San José y Cartago, con el objetivo de hacer un primer acercamiento, en un espacio de conversatorio, que permitiera explorar su opinión en cuanto a los servicios que brinda la universidad en las dimensiones a estudiar.

- Se realizó un borrador de la encuesta que fue revisado en primera instancia por dos personas expertas externas a la UNED con formación en el área educativa y de administración.

- Se realizaron los ajustes solicitados por las personas expertas externas y se procedió a enviar el borrador de encuesta, a modo de prueba piloto del instrumento, a un sujeto graduado de la UNED en cada una de las seis regiones, con el fin de conocer su opinión como exestudiante de la universidad y realizar ajustes.

- Se realizó una primera aplicación de la encuesta a 171 personas (se determinó este número considerando el triple de la cantidad total de ítems), para evaluar su confiabilidad. Para la aplicación de las encuestas se capacitó a un sujeto colaborador por cada una de las 6 regiones que componen las distribuciones de centros universitarios de la UNED.

- Se calculó con las respuestas de las 171 encuestas obtenidas el alfa de Cronbach y medidas descriptivas entre ellas moda y frecuencias. Esto permitió conocer un poco el panorama a investigar.

- Se realizaron los ajustes al instrumento en cuanto a la redacción y corrección de aquellos ítems para los que el estudiantado manifestó dudas. 
http://doi.org/10.15359/ree.25-2.18

http://www.una.ac.cr/educare

educare@una.ac.cr

- Se montó la encuesta en un programa gratuito denominado formularios Google en línea, lo cual permitió generar un link con el que el estudiantado podía acceder desde cualquier dispositivo a la encuesta.

- Se rastreó, mediante correo electrónico, al estudiantado que por algún motivo no llenó la encuesta en su totalidad y por medio de llamadas telefónicas se completó esta.

- Se decodificaron los resultados de la encuesta para el análisis respectivo, se calculó el alfa de Cronbach.

Los datos se procesaron por medio del programa SPSS, para posteriormente realizar el análisis de los resultados.

A partir de estos datos, como ya se mencionó, se calculó el alfa de Cronbach que arrojó un resultado de 0,886 en base a elementos analizados, los que corresponden solo a las variables institucionales, académicas y personales, ya que las variables de identificación no se han incluido en el cálculo de la confiabilidad del instrumento (ver Tabla 2).

Tabla 2: Resultados de alfa de Crombach

Alfa de Cronbach considerando los ítems de los factores institucionales, académicos y personales

$\mathrm{N}$ de elementos

0,886

42

Nota: Elaboración propia.

\section{Resultados, análisis y discusión}

En relación con las variables de identificación la muestra se caracteriza de la siguiente manera:

La muestra en estudio estuvo conformada por 300 mujeres $(71,77 \%)$ y 118 hombres (28,23\%).

Las edades se agruparon por rangos de intervalos, los resultados obtenidos fueron: 109 estudiantes poseían entre $17-20$ años, lo cual representa un $26,08 \%$ de la muestra; 238 se encontraban entre $21-30$ años, representando un $56,94 \%$, este el rango donde se concentra la mayor cantidad de estudiantes que participaron de este estudio; 62 entre 3140 años, que corresponde a un 14,83\% de la muestra; 8 entre $41-50$ años, constituye un $1,91 \%$ y finalmente 1 persona con 51 o más años que en comparación equivale a un 0,24\% del total de la muestra (ver Figura 4). 
http://doi.org/10.15359/ree.25-2.18

http://www.una.ac.cr/educare educare@una.ac.cr

Figura 4: Porcentaje de estudiantes por rango de edad que conformaron la muestra en estudio

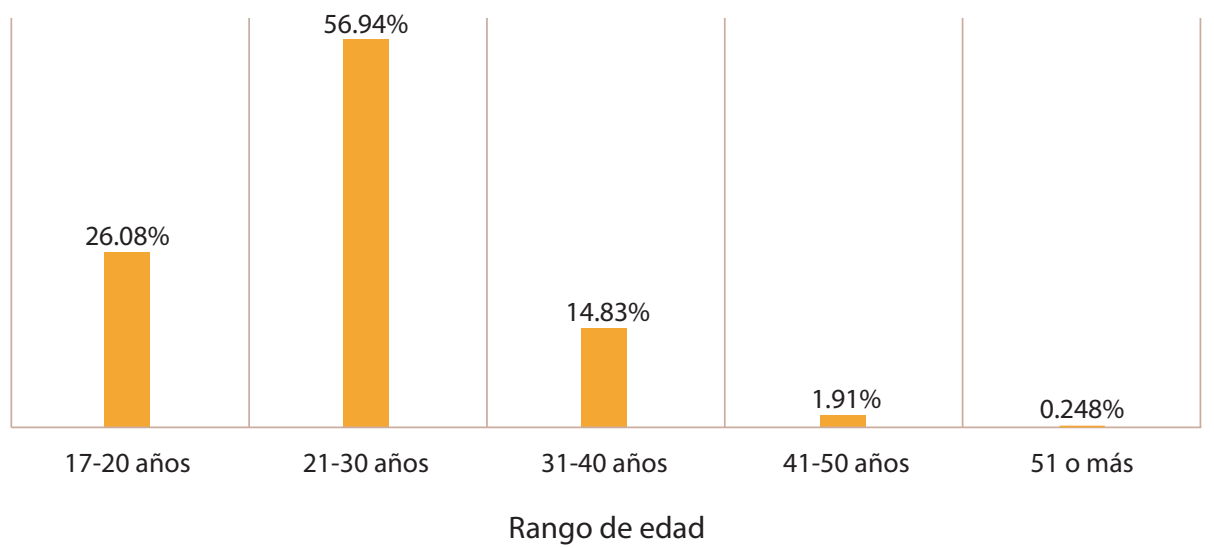

Nota: Elaboración propia.

Al comparar las variables de sexo con los rangos de edad se obtuvo que $38,28 \%$ de la muestra son mujeres con rangos de edad de 21 a 30 años, seguido por mujeres de 17 a 20 años $(21,77 \%)$, el tercer valor es alcanzado por hombres son un rango de 21 a 30 años equivalente a un $18,66 \%$ de la muestra total.

Se consultó al estudiantado para conocer cuántos poseían acceso al servicio de internet en su casa o lugar de residencia, de lo cual resultaron 327 estudiantes, equivalente al 78,23\% de la muestra, que sí poseen y 91 estudiantes, correspondiente al 21,77\% de la muestra, no cuentan con el servicio.

En cuanto a la percepción del estudiantado sobre el estrés que genera el proceso de enseñanza en la UNED se obtuvo que el $52,87 \%$ se siente estresado, el 38,52\% siente un poco de estrés y tan solo el 8,61\% expresa que no se encuentra afectado por este factor.

Se consultó a la muestra en estudio sobre, si alguna vez han pensado en abandonar los estudios en la UNED, y mencionaron 221 estudiantes (52,87\%) que no, 94 (22,49\%) que sí y 79 $(18,90 \%)$ que al menos una vez han analizado la posibilidad de optar por la deserción.

En lo que respecta al nivel de satisfacción con la carrera en la que el estudiantado está inscrito, se observa que en la actualidad 318 estudiantes (76,08\%) se manifiestan satisfacción, $83(19,86 \%)$ un poco y $17(4.07 \%)$ insatisfacción.

De 418 estudiantes que conformaron la muestra en estudio, el 8,61\% espera poder culminar sus estudios en un periodo de 1 año o menos, el 39,00\% espera culminar entre 2 a 3 años, por otro lado, el 38,76\% estima una duración de 4 a 5 años como mínimo y el 13,64\% considera que le falta más de 6 años para graduarse. 
http://doi.org/10.15359/ree.25-2.18

http://www.una.ac.cr/educare

educare@una.ac.cr

Respecto de los resultados de la perspectiva estudiantil y su impacto en el rendimiento académico acerca de las variables institucionales, se determinó:

- Sobre la infraestructura de las instalaciones de la UNED, el 48,60\% percibe que le beneficia mucho, 12,70\% le beneficia poco, 11,00\% no le afecta ni le beneficia, $21,10 \%$ le afecta poco y $6,70 \%$ le afecta mucho. De conformidad con los resultados obtenidos, un alto porcentaje de la muestra expresa que la infraestructura de los centros universitarios beneficia su rendimiento académico.

- En cuanto a los horarios de atención, se determinó que el 50\% percibe que le beneficia mucho, $10,5 \%$ le beneficia poco, $19,00 \%$ no le afecta ni les beneficia, $14,1 \%$ le afecta poco y $0,6 \%$ le afecta mucho. Más del $60 \%$ del estudiantado considera beneficiosos los horarios de atención actual de los centros universitarios para su rendimiento académico.

- En lo correspondiente a las tutorías presenciales se determinó que el $36,1 \%$ percibe que le beneficia mucho, 13,9\% le beneficia poco, $18,00 \%$ no le afecta ni le beneficia, $22,7 \%$ le afecta poco y $8,9 \%$ le afecta mucho.

- En relación con la accesibilidad a internet en el centro universitario se obtuvieron los siguientes resultados: $34,2 \%$ percibe que le beneficia mucho, 15,8\% le beneficia poco, $26,6 \%$ no le afecta ni les beneficia, $13,2 \%$ le afecta poco y $10,3 \%$ Is afecta mucho.

- Referente al factor de accesibilidad a los laboratorios que utilizan en los cursos de carrera el estudiantado, el 49,0\% perciben que le beneficia mucho, 11,7\% les beneficia poco, $13,6 \%$ no le afecta ni les beneficia, $11,5 \%$ le afecta poco y $14,1 \%$ le afecta mucho.

- Respecto a la accesibilidad de horarios de los laboratorios de informática para uso del estudiantado se determinó que el 59,3\% percibe que les beneficia mucho, 12,0\% le beneficia poco, $11,2 \%$ no le afecta ni le beneficia, $10 \%$ le afecta poco y $7,4 \%$ le afecta mucho.

- Acerca del horario en el que se imparten las tutorías se determinó que el 23,0\% percibe que le beneficia mucho, $17,2 \%$ le beneficia poco, $21,5 \%$ no le afecta ni le beneficia, $23,2 \%$ le afecta poco y $15,1 \%$ le afecta mucho.

- En consideración con la ubicación territorial donde se imparten las tutorías se determinó que el $30,9 \%$ percibe que le beneficia mucho, $14,4 \%$ le beneficia poco, $15,6 \%$ no le afecta ni les beneficia, $17,9 \%$ le afecta poco y $21,3 \%$ le afecta mucho.

- En cuanto a la metodología presencial de la UNED para los procesos de entrega de tareas se determinó que el 28,7\% percibe que le beneficia mucho, 18,2\% le beneficia poco, $17,5 \%$ no le afecta ni le beneficia, $23,9 \%$ le afecta poco y $11,7 \%$ le afecta mucho.

- El ítem sobre la disponibilidad de cursos abiertos por cuatrimestre determinó que el $30,1 \%$ percibe que le beneficia mucho, $12,7 \%$ le beneficia poco, $12,7 \%$ no le afecta ni le beneficia, $22,2 \%$ le afecta poco y $22,2 \%$ le afecta mucho. 
http://doi.org/10.15359/ree.25-2.18

- La consulta sobre el lugar donde se aplican los exámenes indicó que el 33,0\% percibe que le beneficia mucho, $12,7 \%$ le beneficia poco, $24,4 \%$ no le afecta ni le beneficia, $20,6 \%$ le afecta poco y $9,3 \%$ le afecta mucho.

- La muestra respondió, en cuanto al cronograma establecido por la UNED para la aplicación de exámenes, que el 28,9\% percibe que le beneficia mucho, para el 11,0\% le beneficia poco, para el 39,7\% no le afecta ni le beneficia, para el 16,7\% le afecta poco y para el 3,6\% le afecta mucho.

- Respecto al sistema de matrícula en línea de la UNED se determinó que el 16,3\% percibe que le beneficia mucho, $13,6 \%$ le beneficia poco, 56,9\% no le afecta ni le beneficia, $8,9 \%$ le afecta poco y $4,3 \%$ le afecta mucho.

- Sobre los periodos cuatrimestrales de la UNED se determinó que el 28,7\% percibe que le beneficia mucho, $17,2 \%$ le beneficia poco, $33,0 \%$ no le afecta ni le beneficia, $16,7 \%$ le afecta poco y $4.3 \%$ le afecta mucho.

- Concerniente al horario de atención disponible por las cátedras se determinó que el $38,5 \%$ percibe que le beneficia mucho, $12,0 \%$ le beneficia poco, $15,8 \%$ no le afecta ni le beneficia, $21,1 \%$ le afecta poco y $12,7 \%$ le afecta mucho.

- Respecto a la información contenida en la página web oficial de la UNED, el 24,2\% de estudiantes percibe que le beneficia mucho, $12,0 \%$ le beneficia poco, $15,8 \%$ no le afecta ni les beneficia, $21,1 \%$ le afecta poco y $12,7 \%$ le afecta mucho.

- Sobre la plataforma estudiantil de la UNED, el 17,5\% de estudiantes indica que le beneficia mucho, $14,8 \%$ le beneficia poco, $53,1 \%$ no le afecta ni le beneficia, $12,0 \%$ le afecta poco y $2,6 \%$ le afecta mucho.

- En relación con el sistema de estudio a distancia que les ofrece la UNED, el 17,5\% determina que le beneficia mucho, $14,8 \%$ le beneficia poco, $53,0 \%$ no le afecta ni le beneficia, $12,0 \%$ le afecta poco y $2,6 \%$ le afecta mucho.

- A nivel general, la variable de accesibilidad de horarios de los laboratorios de informática para uso del estudiantado obtuvo el porcentaje más alto correspondiente a un $59,30 \%$ de la población que considera que esta variable le beneficia mucho en su rendimiento académico.

- En cuanto a la variable de disponibilidad de cursos abiertos por cuatrimestre 22, 20\% de la población de estudiantes considera que esta le afecta mucho en su rendimiento académico, este dato corresponde a uno de los principales resultados negativos que necesita una atención oportuna. 
http://doi.org/10.15359/ree.25-2.18

http://www.una.ac.cr/educare

educare@una.ac.cr

Al realizar un análisis de las 18 variables en estudios que contemplaron la sesión clasificada como institucionales se logró determinar, de acuerdo con el promedio de los resultados, que el porcentaje más alto equivalente a un 33,03\% de la población en estudio determina que percibe que las variables institucionales le benefician mucho en su rendimiento académico y tan solo un $9,23 \%$ que le afecta mucho (ver Tabla 3).

Tabla 3: Resultados porcentuales del promedio de perspectiva estudiantil sobre factores institucionales

\begin{tabular}{cccccc}
\hline $\begin{array}{c}\text { Variable } \\
\text { instituciones }\end{array}$ & $\begin{array}{c}\text { Me afecta } \\
\text { mucho }\end{array}$ & $\begin{array}{c}\text { Me afecta } \\
\text { poco }\end{array}$ & $\begin{array}{c}\text { No me afecta ni } \\
\text { beneficia }\end{array}$ & $\begin{array}{c}\text { Me beneficia } \\
\text { poco }\end{array}$ & $\begin{array}{c}\text { Me beneficia } \\
\text { mucho }\end{array}$ \\
\hline Promedio & $9,23 \%$ & $17,16 \%$ & $25,47 \%$ & $13,73 \%$ & $33,03 \%$ \\
\hline
\end{tabular}

Nota: Elaboración propia.

Al realizar un análisis desde tres posibles perspectivas estudiantiles en cuanto al grado de perjuicio, beneficio o no afectación de las variables institucionales en su rendimiento, un $46,75 \%$ determina que las condiciones actuales que ofrece la UNED benefician su rendimiento académico (ver Tabla 4).

Tabla 4: Resumen de valor porcentual promedio de las variables institucionales en tres áreas generales

\begin{tabular}{cccc}
\hline Variables & Perjudica & No afecta & Beneficia \\
\hline Promedio & $26,39 \%$ & $25,47 \%$ & $46,76 \%$ \\
\hline
\end{tabular}

Nota: Elaboración propia.

En conformidad con los resultados obtenidos, un alto porcentaje de la muestra manifiesta que la infraestructura de los centros universitarios beneficia su rendimiento académico. Este resultado permite justificar la inversión universitaria en infraestructura de los últimos años, pues este es un factor determinante que fundamenta la importancia y el uso adecuado de los recursos otorgados por el Estado.

Actualmente el estudiantado de la UNED considera beneficiosos los horarios de atención de los centros universitarios para su rendimiento académico, lo cual indica que se ha logrado responder a sus necesidades. 
A pesar de que la mayor parte de la muestra en estudio indicó que los espacios físicos donde se llevan a cabo tutorías presenciales son beneficiosos para su rendimiento académico, una parte de estudiantes plantea que este factor afecta su rendimiento, lo cual determina que es necesario realizar una revisión del tipo de espacios donde se desarrollan las tutorías.

En lo correspondiente a la ubicación donde se imparten las tutorías, a pesar de que existe una mayor parte de la muestra que exterioriza que son beneficiosas para el rendimiento académico, otra parte de estudiantes plantea afectación, por lo que es necesario realizar un inventario de infraestructura disponible y demanda actual. En este punto cabe mencionar que muchos de los centros universitarios deben alquilar aulas en colegios y escuelas por la falta de espacio para responder a la cantidad de estudiantado matriculado, esta percepción negativa podría indicar que estos espacios alquilados no se encuentran en ocasiones cercanos al centro universitario.

Como discusión se puede afirmar que según los resultados de los factores institucionales de la UNED, en la perspectiva estudiantil, son beneficios para el rendimiento académico, por tanto, la inversión en esta área por parte de la universidad posee un potencial beneficio, punto importante en la planificación a futuro.

\section{Conclusiones}

Los factores institucionales tales como: tipo de sistema de matrícula (matri-web), plataforma utilizada como entorno estudiantil y sistema de estudio a distancia, en la percepción del estudiantado, no influyen ni afectan el rendimiento académico en la UNED.

Por otra parte, el factor institucional de disponibilidad de cursos abiertos por cuatrimestre influye negativamente en el rendimiento académico de estudiantes de la UNED.

Los otros factores institucionales indagados como: infraestructura del centro universitario, horario de atención en el centro universitario, espacio físico, accesibilidad a internet en el centro universitario, accesibilidad (lugar) de los laboratorios para cursos de la carrera, accesibilidad de horarios de los laboratorios informáticos, horario en el que se imparten las tutorías, lugar donde se imparten las tutorías obligatorias, metodología presencial para entrega de tareas, lugar donde se aplican los exámenes, cronograma de aplicación de exámenes (fines de semana), periodos lectivos cuatrimestrales, horario de atención de los encargados de cátedra, información contenida en la página web influyen positivamente en el rendimiento académico de estudiantes de la UNED. 
http://doi.org/10.15359/ree.25-2.18

http://www.una.ac.cr/educare

educare@una.ac.cr

Los resultados obtenidos constituyen un aporte original que pueden servir de reflexión para las autoridades de la Universidad Estatal a Distancia de Costa Rica, y constituye un paso en los procesos de evaluación de la calidad de la UNED. En este sentido, la perspectiva estudiantil puede dar a conocer a la institución los procesos que debe mejorar y aquellos que debe cambiar.

En cuanto al aporte a la comunidad científica, se establece que se ha desarrollado una investigación en un contexto a distancia, lo que constituye un antecedente para futuras investigaciones en otras universidades con este mismo formato, ya que son escasos los estudios realizados en este ámbito.

Cabe aclarar que los resultados antes mencionados son representativos de las seis regiones geográficas de estudio entre ellas: región Chorotega, Huertar Norte, Pacífico Central, Brunca, Atlántica y Central; logra, así, una cobertura de todas las sedes de la UNED. A partir de estos resultados, se espera que desde los decisores puedan tomarse las medidas que permitan mantener la calidad de los factores identificados como influyentes positivamente en el rendimiento académico y, al mismo tiempo, fortalecer aquellas variables identificadas como obstaculizadoras o que no contribuyen con el éxito en el rendimiento académico.

\section{Declaración de Material complementario}

Este artículo tiene disponible, como material complementario:

-La versión preprint del artículo en https://doi.org/10.5281/zenodo.4081621

\section{Referencias}

Aguila Cabrera, V. (2005). El concepto calidad en la educación universitaria: Clave para el logro de la competitividad institucional. Revista Iberoamericana de Educación, 36(12), 1-7. https:// rieoei.org/RIE/article/view/2886

Carpio Malavasi, L. G. y Parreaguirre Camacho, J. C. (2017). Universidad Estatal a Distancia. Informe de labores 2017. EUNED. https://www.uned.ac.cr/viplan/images/cppi/Informe_Labores UNED 2017.pdf

Chilca Alva, M. L. (2017). Autoestima, hábitos de estudio y rendimiento académico en estudiantes universitarios. Propósitos y Representaciones 5(1), 71-127. http://dx.doi.org/10.20511/ pyr2017.v5n1.145

de la Cruz, A. (2009). Graduados universitarios lasallistas y calidad educativa. Revista de la Universidad de La Salle, 48, 268-278. https://ciencia.lasalle.edu.co/cgi/viewcontent. cgi?article $=1184 \&$ context $=$ ruls 
http://doi.org/10.15359/ree.25-2.18

http://www.una.ac.cr/educare educare@una.ac.cr

Espinoza, E. (2006). Impacto del maltrato en el rendimiento académico. Revista Electrónica de Investigación Psicoeducativa, 4(9), 221-238. http://repositorio.ual.es/bitstream/ handle/10835/638/Art 9 64.pdf? sequence=1\&isAllowed $=\mathrm{y}$

Figueroa Retana, L. F. y Gatgens Gutiérrez, D. (2018). Anuario estadístico 2017. UNED. https:// www.uned.ac.cr/viplan/images/ciei/ANUARIO_2017/Anuario_2017_versi\%C3\%B3n final 19-05-18.pdf

Garbanzo Vargas, G. M. (2007). Factores asociados al rendimiento académico en estudiantes universitarios, una reflexión desde la calidad de la educación superior pública. Revista Educación, 31(1), 43-63. https://doi.org/10.15517/revedu.v31i1.1252

Marchesi, A. (2000). Un sistema de indicadores de desigualdad educativa. Revista lberoamericana de Educación, 23, 135-163. https://doi.org/10.35362/rie2301009

OrozcoGuzmán,J.C.(2016). Hacia una nueva significación deldiscursosobreacreditaciónycalidad de la educación en los colegios públicos de Bogotá. Revista de la Universidad de La Salle, 69, 13-47. https://ciencia.lasalle.edu.co/cgi/viewcontent.cgi?article=1471\&context=ruls

Pineda Ledezma, O. B. y Alcántara Galdámez, N. J. (2017). Hábitos de estudio y rendimiento académico en estudiantes universitarios. Innovare: Revista de Ciencia y Tecnología, 6(2)1934. https://doi.org/10.5377/innovare.v6i2.5569

Pozón López, J. R. (2014). Los estudiantes universitarios ante las actividades extracurriculares. Anduli, 13, 137-150. https://idus.us.es/handle/11441/34934

Rojas, M. (2009). El abandono de los estudios: Deserción y decepción de la juventud. Hologramática, 4(10), 75-94. http://cienciared.com.ar/ra/usr/3/467/hologramatica n10 vol4pp75 94.pdf

Sánchez Herencia, D. (2017). Factores personales que influyen en el rendimiento académico de los estudiantes de la carrera profesional de medicina veterinaria de la Universidad Nacional de San Antonio Abad de Cusco [Tesis de especialización]. Universidad Nacional del Altiplazno, Puno, Perú. http://repositorio.unap.edu.pe/handle/UNAP/8240

Vázquez, C. M., Cavallo, M. A., Aparicio, S. N., Muñoz, B. L., Robson, C. M., Ruiz, L. I., Secreto, M. F., Sepliarsky, P. A. y Escobar, M. E. (2012). Factores de impacto en el rendimiento académico universitario. Un estudio a partir de las percepciones de los estudiantes. Decimoséptimas Jornadas "Investigaciones en la facultad" de Ciencias Económicas y Estadística. Universidad Nacional de Rosario, Santa Fe, Argentina. http://www.fcecon.unr.edu.ar/web-nueva/sites/ default/files/u16/Decimocuartas/vazquez c factores de impacto en el rendimiento academico.pdf 
http://doi.org/10.15359/ree.25-2.18

http://www.una.ac.cr/educare

educare@una.ac.cr

\section{Apéndice $A$}

Tabla 1A: Resumen de valor porcentual promedio de las variables institucionales

\begin{tabular}{|c|c|c|c|c|c|}
\hline Variable instituciones & $\begin{array}{l}\text { Me afecta } \\
\text { Mucho }\end{array}$ & $\begin{array}{l}\text { Me afecta } \\
\text { poco }\end{array}$ & $\begin{array}{l}\text { No me afecta } \\
\text { ni beneficia }\end{array}$ & $\begin{array}{l}\text { Me beneficia } \\
\text { Poco }\end{array}$ & $\begin{array}{l}\text { Me beneficia } \\
\text { mucho }\end{array}$ \\
\hline Infraestructura del Centro Universitario & $6,60 \%$ & $21,10 \%$ & $11,00 \%$ & $12,70 \%$ & $48,60 \%$ \\
\hline Horario de atención en el Centro Universitario & $0,60 \%$ & $14,10 \%$ & $19,40 \%$ & $10,50 \%$ & $55,40 \%$ \\
\hline Espacio físico & $8,90 \%$ & $22,70 \%$ & $18,40 \%$ & $13,90 \%$ & $36,10 \%$ \\
\hline $\begin{array}{l}\text { Accesibilidad a internet en el Centro } \\
\text { Universitario }\end{array}$ & $10,30 \%$ & $13,20 \%$ & $26,50 \%$ & $15,80 \%$ & $34,20 \%$ \\
\hline $\begin{array}{l}\text { Accesibilidad (lugar) de los laboratorios } \\
\text { para cursos de la carrera. }\end{array}$ & $14,10 \%$ & $11,50 \%$ & $13,60 \%$ & $11,80 \%$ & $49,00 \%$ \\
\hline $\begin{array}{l}\text { Accesibilidad de horarios de los } \\
\text { laboratorios informáticos }\end{array}$ & $7,50 \%$ & $10,00 \%$ & $11,20 \%$ & $12,00 \%$ & $59,30 \%$ \\
\hline Horario en el que se imparten las tutorías & $15,10 \%$ & $23,20 \%$ & $21,50 \%$ & $17,20 \%$ & $23,00 \%$ \\
\hline $\begin{array}{l}\text { Lugar donde se imparten las tutorías } \\
\text { obligatorias }\end{array}$ & $21,30 \%$ & $17,90 \%$ & $15,60 \%$ & $14,40 \%$ & $30,80 \%$ \\
\hline Metodología presencial para entrega de tareas & $11,70 \%$ & $23,90 \%$ & $17,50 \%$ & $18,20 \%$ & $28,70 \%$ \\
\hline $\begin{array}{l}\text { Disponibilidad de cursos abiertos por } \\
\text { cuatrimestre }\end{array}$ & $22,30 \%$ & $22,20 \%$ & $12,70 \%$ & $12,70 \%$ & $30,10 \%$ \\
\hline Lugar donde se aplican los exámenes & $9,30 \%$ & $20,60 \%$ & $24,40 \%$ & $12,70 \%$ & $33,00 \%$ \\
\hline $\begin{array}{l}\text { Cronograma de aplicación de exámenes } \\
\text { (fines de semana) }\end{array}$ & $3,60 \%$ & $16,70 \%$ & $39,70 \%$ & $11,00 \%$ & $29,00 \%$ \\
\hline Tipo de sistema de matrícula (matri-web) & $4,30 \%$ & $8,90 \%$ & $56,90 \%$ & $13,60 \%$ & $16,30 \%$ \\
\hline Periodos lectivos cuatrimestrales & $4,30 \%$ & $16,70 \%$ & $33,00 \%$ & $17,20 \%$ & $28,80 \%$ \\
\hline $\begin{array}{l}\text { Horario de atención del personal } \\
\text { encargado de cátedra }\end{array}$ & $12,70 \%$ & $21,00 \%$ & $15,80 \%$ & $12,00 \%$ & $38,50 \%$ \\
\hline Información contenida en la página web & $3,60 \%$ & $12,00 \%$ & $41,10 \%$ & $19,10 \%$ & $24,20 \%$ \\
\hline Plataforma utilizada como entorno estudiantil. & $2,60 \%$ & $12,00 \%$ & $53,10 \%$ & $14,80 \%$ & $17,50 \%$ \\
\hline Sistema de estudio a distancia. & $5,50 \%$ & $12,70 \%$ & $52,20 \%$ & $17,50 \%$ & $12,10 \%$ \\
\hline
\end{tabular}

Nota: Resultados porcentuales del promedio de perspectiva estudiantil sobre factores institucionales. Elaboración propia.

\begin{tabular}{r}
\hline $20 \quad$ José Alejandro Echeverría-Ramírez y Claudia Mazzitelli \\
Los artículos de la Revista Electrónica Educare del Centro de Investigación y Docencia en Educación de la Universidad Nacional, Costa Rica, se comparten bajo términos de la Licencia \\
Creative Commons: Reconocimiento, No Comercial, Sin Obra Derivada 3.0 Costa Rica. Las autorizaciones adicionales a las aquí delimitadas se pueden obtener en el correo: educare@una.cr $@$ CFY No ND
\end{tabular}

